\title{
NEW SUPERVISORY CONTROL USING CONTROL-RELEVANT SWITCHING
}

\author{
Tae-Woong Yoon*, Jung-Su Kim ** \\ * Dept. of Electrical Engineering. Korea University, \\ Anam-dong 5-ga Seongbuk-gu 136-713, Seoul, Korea, \\ twy@korea.ac.kr, jsk@cello.korea.ac.kr \\ ** ASRI, School of Electrical Engineering and Computer \\ Science \#060 Seoul National University Kwanak PO Box \\ 34, Seoul, 151-600, Korea, jskim@acorn.snu.ac.kr
}

\begin{abstract}
This paper presents a new supervisory control scheme, which is based on a control-relevant switching logic. Unlike most of the existing switching methods considering only estimator performance, the proposed scheme takes both estimator and controller performance into account. As an index to the controller performance, an iISS (integral-Input-to-State Stability) Lyapunov function is employed; it is ensured that the Lyapunov function satisfies a certain inequality. This Lyapunov-based switching is then coupled to the state-dependent dwell-time switching developed recently, and the state of the uncertain plant is shown to converge asymptotically. Copyright(C)2005 IFAC.
\end{abstract}

Keywords: supervisory control, iISS Lyapunov function, dwell-time switching

\section{INTRODUCTION}

Supervisory control employs logic-based switching for adaptation, instead of continuous tuning of parameters as in conventional adaptive control. This type of switching-based supervisory control scheme consists of the following subsystems: an uncertain plant to be controlled, a bank of estimators, a bank of controllers, a performance monitoring signal generator, and a switching logic. See (Morse, 1996; Hespanha and Morse, 1999; Narendra and Xiang, 2000; Liberzon et al., 2001; Hespanha et al., 2002; Hespanha et al., 2003b; De Persis et al., 2004) for a general structure of supervisory control.

Scale independent hysteresis and dwell-time methods are representative of the switching logic for supervisory control; see (Middleton et al., 1988; Hespanha and Morse, 1999; Hespanha et al., 2002; Hespanha et al., 2003a) for the former, and
(Morse, 1996; Borrelli et al., 1999; De Persis et al., 2004; Kim et al., 2004b; Kim et al., 2004a) for the latter. Switching algorithms of both the types are based only on the estimator performance. On the other hand, Lyapunov functions are employed in such switching methods as in (Angeli and Mosca, 2003; Angeli and Mosca, 2004); in these methods, however, an exhaustive search type of algorithm is used, and no estimation performance is considered.

In this paper, we present a new switching logic, which takes both control and estimation performance into account. At every sampling instant, we search for a model corresponding to the smallest monitoring signal. We then decide whether to switch to the resulting model or not by comparing the current value of the iISS (integralInput-to-State Stability) Lyapunov function with its prospective value that would result from the switching; if a certain inequality condition is sat- 
isfied, switching is allowed. We first prove asymptotic convergence of the state of the uncertain plant under the assumption of persistent switching. To deal with the situation where switching stops in a finite time since the switching condition is never satisfied after that time, we further employ a state-dependent dwell-time algorithm together, and force switching to take place every now and then. Note that the dwell-time algorithm devised here is slightly different from the recent ones in (De Persis et al., 2004; Kim et al., 2004a). Finally we show that asymptotic convergence is ensured, without assuming persistent switching, by the proposed supervisory control scheme resulting from the combination of the Lyapunovfunction-based switching and the state-dependent dwell-time switching.

\section{SUPERVISORY CONTROL}

The supervisory control architecture under consideration is depicted in Fig. 1. For more details of the general structure of supervisory control, see (Morse, 1996; Hespanha and Morse, 1999; Liberzon et al., 2001; Hespanha et al., 2002; De Persis et al., 2004). As seen in Fig. 1, the overall structure is similar to that of conventional adaptive control. However, the difference is that the adaptation is carried out via switching in the supervisory control. This is in sharp contrast with conventional adaptive control in which adaptation is based on continuous tuning.

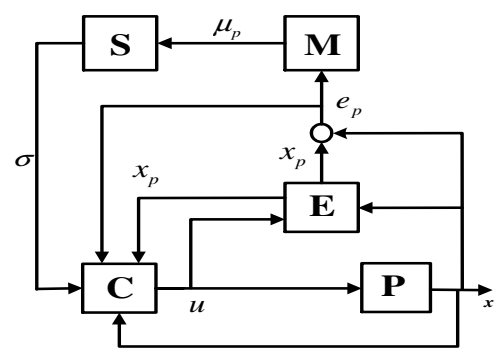

Fig. 1. Supervisory control architecture

\subsection{The uncertain plant and the multi-estimator}

The block $\mathbf{P}$ in Figure 1 is the uncertain plant to be controlled, and is described by

$$
x(k+1)=f_{p^{*}}(x(k), u(k))
$$

where $x \in \mathbb{R}^{n}$ is the state, and $u \in \mathbb{R}^{m}$ the control input. It is assumed that $f_{p^{*}}$ is unknown but is a member of a known set $\left\{f_{p}: p \in \mathcal{P}\right\}$ with $\mathcal{P}$ being the index set; in other words, $p^{*}$ is an unknown member of the known index set $\mathcal{P}$. For simplicity, $\mathcal{P}$ is assumed to be finite. The multi-estimator $\mathbf{E}$ is a bank of estimators; the estimator for the $p$-th model is written as

$$
x_{p}(k+1)=A_{E}\left(x_{p}(k)-x(k)\right)+f_{p}(x(k), u(k))
$$

where $A_{E}$ is a Hurwitz matrix. The estimation error is defined by

$$
e_{p}(k)=x_{p}(k)-x(k), \quad p \in \mathcal{P} .
$$

Note that the error dynamics associated with the correct model is given by

$$
e_{p^{*}}(k+1)=A_{E} e_{p^{*}}(k) .
$$

Therefore, $e_{p^{*}}(k)$ decays exponentially, i.e.

$$
\left\|e_{p^{*}}(k)\right\| \leq C_{1} \nu^{* k}
$$

where $C_{1}>0$ and $\nu^{*}=\max \left|\lambda\left(A_{E}\right)\right|$ with $\lambda(\cdot)$ denoting the eigenvalues.

\subsection{The multi-controller}

The multi-controller $\mathbf{C}$ is assumed to be of the form

$$
u_{p}(k)=k_{p}(x(k)) .
$$

We now write, in terms of the $p$-th model and the estimation error, the uncertain plant as follows:

$$
\begin{aligned}
x(k+1) & =f_{p}(x(k), u(k))-\left(e_{p}(k+1)-A_{E} e_{p}(k)\right) \\
& =: f_{p}(x(k), u(k))-d_{p}(k) .
\end{aligned}
$$

The closed-loop consisting of the control law (6) using the $p$-th model and the plant (7) is referred to as the injected system, and is written as

$$
\begin{aligned}
x(k+1) & =f_{p}\left(x(k), k_{p}(x(k))\right)-d_{p}(k) \\
& =: A_{p}\left(x(k), d_{p}(k)\right) .
\end{aligned}
$$

The multi-controller is assumed to be such that the injected system (8) is iISS (integral Input-toState Stable) when $d_{p}$ is considered the external input.

Assumption 1. For each $p \in \mathcal{P}$, there exist $\alpha_{p}, \gamma_{p} \in \mathcal{K}_{\infty}, \beta_{p} \in \mathcal{K} \mathcal{L}$ such that

$$
\alpha_{p}(\|x(k)\|) \leq \beta_{p}(\|x(0)\|, k)+\sum_{i=0}^{k-1} \gamma_{p}(\|d(i)\|) .
$$

As discussed in (Angeli, 1999; Sontag, 1998), this iISS property is equivalent to the existence of an iISS Lyapunov function $V_{p}: \mathbb{R}^{n} \rightarrow \mathbb{R}_{>0}$ such that, for $\alpha_{p 1}, \alpha_{p 2}, \gamma_{p} \in \mathcal{K}_{\infty}$ and a positive function $\rho_{p}$, $V_{p}$ satisfies

$$
\alpha_{p 1}(\|x\|) \leq V_{p}(x) \leq \alpha_{p 2}(\|x\|),
$$

and

$$
V_{p}\left(A_{p}\left(x, d_{p}\right)\right)-V_{p}(x) \leq-\rho_{p}(\|x\|)+\gamma_{p}\left(\left\|d_{p}\right\|\right) .
$$

In view of the inequality in (5), we also make the following assumption.

Assumption 2. There exists a positive constant $\eta^{*} \in(0,1)$ such that

$$
\gamma_{p^{*}}\left(\left\|e_{p^{*}}(k)\right\|\right) \leq C_{2} \eta^{* k}
$$

where $C_{2}$ is a positive constant.

Note that Assumption 2 is guaranteed to hold if $\gamma_{p^{*}}$ is a polynomial owing to (5). 


\subsection{The performance monitoring signal generator}

The performance monitoring signal $\mu_{p}$ associated with each $p \in \mathcal{P}$ is defined by

$$
\mu_{p}(k)=\eta \mu_{p}(k-1)+\gamma_{p}\left(\left\|e_{p}(k)\right\|\right)
$$

with $\eta \in\left(\eta^{*}, 1\right)$, and $\gamma_{p}$ defined in Assumption 1. Note that the input to the monitoring signal generator, i.e., $\gamma_{p}\left(\left\|e_{p}(k)\right\|\right)$ relates to an exponentially weighted version of $\sum_{i=0}^{k} \gamma_{p}\left(\left\|e_{p}(i)\right\|\right)$ in the iISS characterization (9) for the injected system (8). To see this, define

$$
\begin{aligned}
\bar{\mu}_{p}(k) & =\bar{\mu}_{p}(k-1)+\eta^{-k} \gamma_{p}\left(\left\|e_{p}(k)\right\|\right) \\
& =\sum_{i=0}^{k} \eta^{-i} \gamma_{p}\left(\left\|e_{p}(i)\right\|\right)
\end{aligned}
$$

Then we have

$$
\begin{aligned}
\bar{\mu}_{p}(k) & =\eta^{-k} \mu_{p}(k), \\
\arg \min _{p \in \mathcal{P}} \mu_{p}(k) & =\arg \min _{p \in \mathcal{P}} \bar{\mu}_{p}(k) .
\end{aligned}
$$

Smallness of a monitoring signal implies that the controller designed using the corresponding model may provide satisfactory performance.

The switching logic $\mathbf{S}$ places in the feedback loop the controller designed using the model from an estimator corresponding to the smallest monitoring signal; the next section focuses on the switching logic proposed in this paper.

\section{CONTROL-RELEVANT SWITCHING}

The output of the switching logic is denoted by $\sigma(k) \in \mathcal{P}$, the value of which is the index of the model selected by the switching logic at time $k$. Here we discuss how to select this piecewise constant signal.

\subsection{Switching using Lyapunov functions}

We present a new switching logic using the iISS Lyapunov functions in (10) and (11). The proposed algorithm is referred to as $\mathbb{S}_{L F}$, and is given as follows:

\section{Algorithm $\mathbb{S}_{L F}$ :}

1. Initialize $\sigma(k)$; initialize $s(k):=0$

2 . Find the best model

$$
q:=\arg \min _{p \in \mathcal{P}} \bar{\mu}_{p}(k)
$$

3. If $\sigma(k)=q$, then $s(k):=0$ else if

$$
V_{q}(x(k))-V_{\sigma}(x(k)) \leq \theta s(k) \quad(\theta<1)
$$

then $\sigma(k):=q$ and $s(k):=0$
4. Compute and apply the control

$$
u(k):=u_{\sigma}(k)
$$

5. $s(k+1):=s(k)+\rho_{\sigma}(\|x(k)\|)$

6 . Go to step 2 at the next time $(k:=k+1)$.

Switching is allowed to take place in $\mathbb{S}_{L F}$ when two conditions are met: firstly there should be a better model leading to the minimum value of the monitoring signal, and secondly the inequality in (17) should hold. In other words, switching is not allowed even when there is a better model, if use of this new model increases the value of the Lyapunov function in such a way that violates the condition given in (17). Checking the two conditions implies that both estimator and control performance is considered in $\mathbb{S}_{L F}$. As result of employing the switching logic $\mathbb{S}_{L F}$, we have the following:

Theorem 1. Consider the supervisory control system consisting of the plant (1), the multi-estimator (2), the multi-controller (6), the performance monitoring signal generator (13), and the switching logic $\mathbb{S}_{L F}$. Suppose that Assumption 1 holds and therefore the inequalities in (10) and (11) are satisfied. Then, for all $k>k_{0}$, we have

$$
\begin{aligned}
& V(x(k))-V\left(x\left(k_{0}\right)\right) \\
& \leq \sum_{l=k_{0}}^{k-1}\left[-(1-\theta) \rho_{\sigma(l)}(\|x(l)\|)+\gamma_{\sigma(l)}\left(\left\|d_{\sigma(l)}(l)\right\|\right)\right],
\end{aligned}
$$

where $V(x(k))=V_{\sigma(k)}(x(k))$.

Proof: This theorem follows in a straightforward manner from the inequalities in (11) and (17), and thus the detailed procedure is not given here.

Remark 2. As Theorem 1 shows, the switching condition given in (17) is used to guarantee the inequality in (18). However, the inequality in (18) alone does not lead to stability since $\gamma_{\sigma}\left(\left\|d_{\sigma}(k)\right\|\right)$ may not be summable. As is shown in the next section, if Assumption 2 holds, and if switching persists, then (18) results in convergence of the state $x(k)$ to zero.

To deal with the situation where switching stops in a finite time since (17) is never satisfied after that time, we further employ a state-dependent dwell-time algorithm together so as to force switching to take place every now and then.

\subsection{Combining $\mathbb{S}_{L F}$ with dwell-time switching}

A dwell time is a lower bound for the difference between any consecutive switching instants; in other words, switching is allowed after waiting for the dwell time. For nonlinear systems, the dwell 
time needs to depend on the state: hence the name 'state-dependent dwell-time switching' (De Persis et al., 2004; Kim et al., 2004a). Here we slightly modify the state-dependent dwell-time algorithm developed in (Kim et al., 2004b; Kim et al., 2004a) in order to facilitate the combination with our switching logic proposed above. For the dwell-time switching to be referred to as $\mathbb{S}_{D}$, we first make the following assumption.

Assumption 3. The injected system in (8) is locally exponentially stable when $d_{p}=0$. In other words, for each $p \in \mathcal{P}$, there exist a function $W_{p}(x): \mathbb{R}^{n} \rightarrow \mathbb{R}_{\geq 0}$, and positive real numbers $a_{1}, a_{2}, a_{3}$ and $\bar{s}$ such that for $\|x\| \leq \bar{s}$

$$
\begin{gathered}
a_{1}\|x\|^{2} \leq W_{p}(x) \leq a_{2}\|x\|^{2}, \\
W_{p}\left(A_{p}(x, 0)\right)-W_{p}(x) \leq-a_{3}\|x\|^{2} .
\end{gathered}
$$

As there are two switching logics involved, we use two subscripts for switching times to clarify which algorithm causes the switching; Let $k_{i, j}$ denote the switching instant which is due to the $j$-th switching by $\mathbb{S}_{L F}$ in a row after the $i$-th switching by $\mathbb{S}_{D}$.

We now present $\mathbb{S}_{D}$, i.e. the modified dwell-time algorithm. Define first the function $\tau_{\Delta}(\cdot)$ as the smallest integer satisfying

$$
\beta_{p}\left(\left\|x\left(k_{i, j}\right)\right\|, \tau_{\Delta}\left(\left\|x\left(k_{i, j}\right)\right\|\right)\right) \leq \frac{1}{2} \alpha_{p}\left(\frac{1}{\zeta} \| x\left(k_{i, 0} \|\right)\right)
$$

where $\zeta>2$. The state-dependent dwell-time function is then given by

$$
\tau_{D}(r) \geq \begin{cases}\tau_{\Delta}(r), & r \geq \bar{r} \\ \max \left\{\log _{\lambda} \frac{a_{1}}{a_{2}}, \tau_{\Delta}(\bar{r})\right\}, & r<\bar{r}\end{cases}
$$

where $\bar{r}$ is such that

$$
\bar{s}=\alpha^{-1}(2 \beta(\bar{r}, 0)),
$$

$\lambda \in\left[\sqrt{1-\frac{a_{3}}{a_{2}}}, 1\right)$, and $a_{1}, a_{2}, a_{3}$ and $\bar{s}$ are as in Assumption 2.

The two switching logics $\mathbb{S}_{L F}$ and $\mathbb{S}_{D}$ are employed together in the proposed supervisory control scheme; as a result, switching takes place whichever logic allows, thereby leading to more active switching without destroying stability as is shown in the next section. The proposed controlrelevant switching logic is referred to as $\mathbb{S}_{L F} \cup \mathbb{S}_{D}$, and is depicted in Fig. 2.

Remark 3. In $\mathbb{S}_{D}$, switching is not allowed during the dwell time even when there is a better model. Hence the proposed method can be viewed as an improved version of the dwell-time algorithm in that more frequent switching, i.e. more active adaptation is achieved.

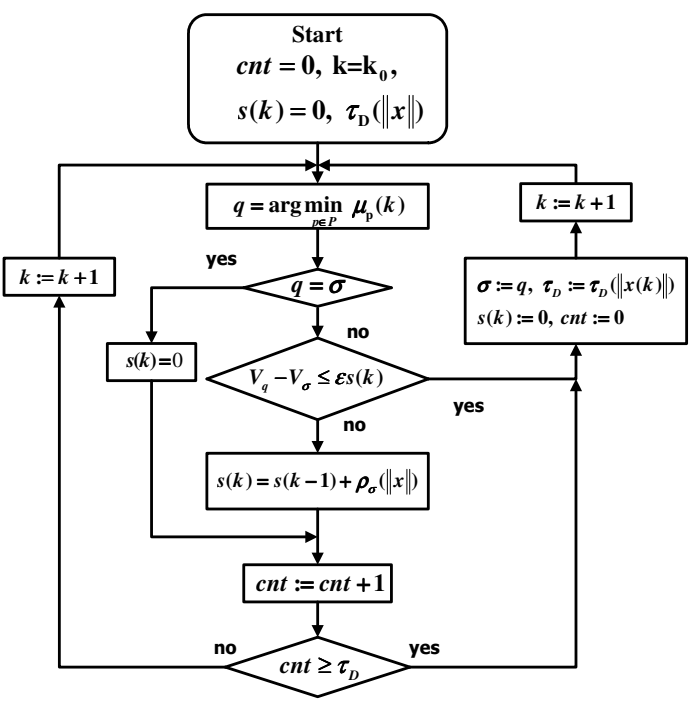

Fig. 2. The proposed switching logic $\mathbb{S}_{L F} \cup \mathbb{S}_{D}$

3.3 Stability of the proposed supervisory control system

Here we prove the closed-loop stability of the proposed supervisory control system. Denote first the value of the switching signal $\sigma$ on $\left[k_{i, j}, k_{i, j+1}\right)$ by $p_{i, j}$, i.e.

$$
\sigma\left(k_{i, j}\right)=\sigma\left(k_{i, j}+1\right)=\cdots=\sigma\left(k_{i, j+1}-1\right)=p_{i, j} .
$$

Regarding the multi-estimator, we first have the following lemma.

Lemma 4. Suppose that Assumption 2, i.e. the inequality in (12) holds. Then there exist a finite switching time $k^{*}$ and a set $\mathcal{P}^{*} \subset \mathcal{P}$ containing $p^{*}$ such that

1. for any switching time $k_{i, j}>k^{*}, \sigma\left(k_{i, j}\right) \in \mathcal{P}^{*}$, 2. for $\sigma(k) \in \mathcal{P}^{*}$

$$
\sum_{k=k_{0}}^{\infty} \gamma_{\sigma(k)}\left(\left\|e_{\sigma(k)}(k)\right\|\right)<\infty, \quad \sum_{k=k_{0}}^{\infty} \gamma_{\sigma(k)}\left(\left\|d_{\sigma(k)}(k)\right\|\right)<\infty .
$$

Proof: It follows from the inequality in (12) and the definition of $\bar{\mu}_{p}(k)$ in (14) that

$$
\lim _{k \rightarrow \infty} \bar{\mu}_{p^{*}}(k)<\infty .
$$

The theorem then results from this inequality; the detailed procedure closely parallels that of the corresponding lemma given in (Morse, 1996), and therefore is not given here.

Theorem 5. Consider the supervisory control system consisting of the plant (1), the multi-estimator (2), the multi-controller (6), the performance monitoring signal generator (13), and the switching logic $\mathbb{S}_{L F}$. Suppose that Assumptions 1 and 2 hold, and that switching takes place persistently. Then, the state of the uncertain plant converges to zero.

Proof. This theorem follows directly from (18) in Theorem 1 and (24) in Lemma 4. 
The persistent switching condition for asymptotic convergence in Theorem 1 can be removed by combining the control-relevant switching $\mathbb{S}_{L F}$ and the modified dwell-dwell time switching $\mathbb{S}_{D}$, i.e., by employing $\mathbb{S}_{L F} \cup \mathbb{S}_{D}$ as follows:

Theorem 6 . Consider the supervisory control system consisting of the plant (1), the multi-estimator (2), the multi-controller (6), the performance monitoring signal generator (13), and the switching logic $\mathbb{S}_{L F} \cup \mathbb{S}_{D}$. Suppose that Assumptions 1, 2, and 3 hold. Then, the state of the uncertain plant converges to zero.

Proof. Note that persistent switching results from $\mathbb{S}_{D}$. If switching persistently takes place only due to either $\mathbb{S}_{L F}$ or $\mathbb{S}_{D}$, then asymptotic convergence results from either Theorem 5 above or from (Kim et al., 2004a). Hence,we consider only the case where both $\mathbb{S}_{L F}$ and $\mathbb{S}_{D}$ become persistently active.

In view of (24) in Lemma 4, for any $\varepsilon>0$, there exists $l^{*}$ satisfying

$$
\sum_{i=k_{l^{*}, 0}}^{\infty} \gamma_{\sigma}\left(\left(\left\|d_{\sigma}(i)\right\|\right) \leq \frac{1}{2} \alpha_{\sigma}\left(\frac{\varepsilon}{\zeta}\right) .\right.
$$

Suppose that $l \geq l^{*}$ and $\left\|x\left(k_{l, 0}\right)\right\| \geq \varepsilon$. Then, letting $k_{l, M_{l}}$ denote the last switching instant due to $\mathbb{S}_{L F}$ before $k_{l+1,0}$, we have

$\alpha_{\sigma}(\|x(k)\|) \leq \beta_{\sigma}\left(\left\|x\left(k_{l, M_{l}}\right)\right\|, k-k_{l, M_{l}}\right)+\sum_{j=k_{l, M_{l}}}^{k-1} \gamma_{\sigma}\left(\left(\left\|d_{\sigma}(j)\right\|\right)\right.$

for $k_{l, M_{l}}+\tau_{D}\left(\left\|x\left(k_{l, M_{l}}\right)\right\|\right) \leq k \leq k_{l+1,0}$. It then follows from the inequalities in (9), (21), and (26) that

$$
\begin{aligned}
& \|x(k)\| \leq \alpha_{\sigma}^{-1}\left(2 \beta_{\sigma}\left(\left\|x\left(k_{l, M_{l}}\right)\right\|, k-k_{l, M_{l}}\right)\right) \\
& +\alpha_{\sigma}^{-1}\left(2 \sum_{j=k_{l, M_{l}}}^{k-1} \gamma_{\sigma}\left(\left(\left\|d_{\sigma}(i)\right\|\right)\right) \leq \frac{1}{\zeta} \cdot\left\|x\left(k_{l, 0}\right)\right\|+\frac{1}{\zeta} \cdot \varepsilon\right. \\
& \leq \frac{1}{\zeta} \cdot\left\|x\left(k_{l, 0}\right)\right\|+\frac{1}{\zeta} \cdot\left\|x\left(k_{l, 0}\right)\right\|=\frac{2}{\zeta} \cdot\left\|x\left(k_{l, 0}\right)\right\| .
\end{aligned}
$$

This, in turn, results in the existence of an index $l^{* *} \geq l^{*}$ such that $\left\|x\left(k_{m, 0}\right)\right\|<\varepsilon$ for all $m \geq l^{* *}$, as assuming $\left\|x\left(k_{m+1,0}\right)\right\| \geq \varepsilon$ leads to the following contradiction

$$
\varepsilon \leq\left\|x\left(k_{m+1,0}\right)\right\| \leq \frac{1}{\zeta}\left\|x\left(k_{m, 0}\right)\right\|+\frac{1}{\zeta} \varepsilon<\frac{2}{\zeta} \varepsilon .
$$

Hence

$$
\lim _{l \rightarrow \infty}\left\|x\left(k_{l, 0}\right)\right\|=0 .
$$

Now consider the values of the state at switching instants by $\mathbb{S}_{L F}$. From equations (10) and (18), we have for $k \in\left(k_{l, 0}, k_{l+1,0}\right)$

$$
\begin{aligned}
& \alpha_{\sigma}(\|x(k)\|) \leq V(x(k)) \\
& \leq\left[V_{\sigma}\left(x\left(k_{l, 0}\right)\right)-(1-\theta) \sum_{j=k_{l, 0}}^{k-1} \rho_{\sigma}(\|x(j)\|)\right]+\sum_{j=k_{l, 0}}^{k-1} \gamma_{\sigma}\left(\left(\left\|d_{\sigma}(j)\right\|\right) .\right.
\end{aligned}
$$

This leads to

$$
\begin{gathered}
\|x(k)\| \leq \alpha_{\sigma}^{-1}\left(2\left[V_{\sigma}\left(x\left(k_{l, 0}\right)\right)-(1-\theta) \sum_{j=k_{l, 0}}^{k-1} \rho_{\sigma}(\|x(j)\|)\right]\right) \\
+\alpha_{\sigma}^{-1}\left(2 \sum_{j=k_{l, 0}}^{k-1} \gamma_{\sigma}\left(\left(\left\|d_{\sigma}(i)\right\|\right)\right)\right. \\
\leq \alpha_{M}\left(2 V_{M}\left(x\left(k_{l, 0}\right)\right)-2(1-\theta) \sum_{j=k_{l, 0}}^{k-1} \rho_{\sigma}(\|x(j)\|)\right) \\
+\alpha_{M}\left(2 \sum_{j=k_{l, 0}}^{k-1} \gamma_{\sigma}\left(\left(\left\|d_{\sigma}(i)\right\|\right)\right)\right. \\
\leq \alpha_{M}\left(2 V_{M}\left(x\left(k_{l, 0}\right)\right)\right)+\alpha_{M}\left(2 \sum_{j=k_{l, 0}}^{k-1}\left\|d_{\sigma}(j)\right\|\right)
\end{gathered}
$$

where

$$
\alpha_{M}(r):=\max _{\sigma \in \mathcal{P}} \alpha_{\sigma}^{-1}(r) \text { and } V_{M}(r):=\max _{\sigma \in \mathcal{P}}\left\{V_{\sigma}(r)\right\} .
$$

Now define $\varepsilon_{1}$ such that $\alpha_{M}\left(2 V_{M}\left(\varepsilon_{1}\right)\right) \leq \frac{1}{2} \varepsilon$. Then there exist indices $i_{1}^{*}$ and $i_{2}^{*}$ such that $\left\|x\left(k_{l, 0}\right)\right\|<$ $\varepsilon_{1}$ for all $l>i_{1}^{*}$ and $\sum_{\tau=k}^{\infty}\left\|d_{\sigma(\tau)}(\tau)\right\|<$ $\alpha_{M}\left(2 V_{M}\left(\varepsilon_{1}\right)\right)$ for all $k>k_{i_{2}^{*}, 0}$. Hence, for all $l>\max \left\{i_{1}^{*}, i_{2}^{*}\right\}$ and $k>k_{l}$,

$$
\begin{aligned}
\|x(k)\| & \leq \alpha_{M}\left(2 V_{M}\left(x\left(k_{l, 0}\right)\right)\right)+\sum_{\tau=k_{i_{2}^{*}, 0}}^{\infty}\left\|d_{\sigma(\tau)}(\tau)\right\| \\
& \leq \alpha_{M}\left(2 V_{M}\left(\varepsilon_{1}\right)\right)+\alpha_{M}\left(2 V_{M}\left(\varepsilon_{1}\right)\right) \\
& \leq \frac{\varepsilon}{2}+\frac{\varepsilon}{2}=\varepsilon .
\end{aligned}
$$

This implies that the sequence of the state at all switching instants converges to zero.

\section{CONCLUSION}

This paper presents a control-relevant switching logic using iISS Lyapunov functions. Unlike most of the existing switching logics, the proposed switching logic considers both estimator and controller performance. The switching logic using the Lyapunov functions is combined with a modified version of the state-dependent dwell-time switching to ensure persistent switching. It is shown that the state of the supervisory control system with the proposed switching logic converges to zero.

\section{REFERENCES}

Angeli, David (1999). Intrinsic robustness of global asymptotic stability. Systems and Control Letters, 38 pp. 297-307.

Angeli, David and Edoardo Mosca (2003). Lyapunov-based switching supervisory control of nonlinear uncertain systems. IEEE Trans. on Auto. Control 47(3) pp. 500-505. 
Angeli, David and Edoardo Mosca (2004). Adaptive switching supervisory control of nonlinear systems with no prior knowledge of noise boundeds. Automatica, Vol. 40 pp. 449-457.

Borrelli, Donato, A. Stephen Morse and Edoardo Mosca (1999). Discrete-time supervisory control of families of two-degrees-of-freedom linear set-point controllers. IEEE Trans. on AC, Vol. 44 pp. $178-181$.

De Persis, Claudio, Raffaella De Santis and A. Stephen Morse (2003). Nonlinear switched systems with state dependent dwell time. Systems and Control Letters, 50(4) pp. 291-302.

De Persis, Claudio, Raffaella De Santis and A. Stephen Morse (2004). Supervisory control with state-dependent dwell-time logic and constraints. Automatica 40, 269-275.

Hespanha, Joao, Daniel Liberzon and A. S. Morse (2002). Supervision of integral-inputto-state stabilizing controllers. Automatica 38(8), 1327-1335.

Hespanha, Joao, Daniel Liberzon and A. S. Morse (2003a). Hysteresis-based switching algorithms for supervisory control of uncertain systems. Automatica, 39(2).

Hespanha, Joao, Daniel Liberzon and A. S. Morse $(2003 b)$. Overcoming the limitations of adaptive control by means of logic-based switching. Systems and Control Letters 49, 2003.

Hespanha, Joao P. and A. Stephen Morse (1999). Certainty equivalence implies detectability. Systems and Control Letters, 36(1) pp. 1-13.

Kim, Jung-Su, Tae-Woong Yoon and Claudio De Persis (2004a). Discrete-time supervisory control of input constrained neutrally stable linear systems via state dependent dwell time switching logic. IFAC Workshop on Adaptation and Learning in Control and Signal Processing, Japan.

Kim, Jung-Su, Tae-Woong Yoon and Claudio De Persis (2004b). State-dependent dwell time switching for discrete-time stable systems. To appear in IEICE TRANS. on Fundamentals, 2004, Temporarily available at http://adaptive.korea.ac.kr/publish/sddt.pdf.

Liberzon, Daniel, Joao Hespanha and A. Stephen Morse (2001). Control using logic and switching. Lecture Note for IEEE CDC 2001, Workshop.

Middleton, Richard H., Graham C. Goodwin, David J. Hill and David Q. Mayne (1988). Design issues in adaptive control. IEEE Trans. on Auto. Contro pp. 50-58.

Morse, A. Stephen (1996). Supervisory control of families of linear set-point controllers-part i:exact matching. IEEE Trans. on Auto. Control pp. 1413-1431.

Narendra, K.S. and Cheng Xiang (2000). Adaptive control of discrete-time systems using multiple models. IEEE Transactions on Automatic Control, 45(9) pp. 1669-1686.

Sontag, Eduardo D. (1998). Comments on integral variants of iss. Systems and Control Letters, vol. 34 pp. $93-100$. 